\title{
Orders of quadratic extensions of number fields
}

\author{
by \\ Jin NAKAGAWA (Joetsu)
}

Let $k$ be a number field of degree $n$ over $\mathbb{Q}$. We denote by $\boldsymbol{o}_{k}, d$ and $E$ the ring of integers of $k$, the discriminant of $k$ and the group of units of $k$, respectively. Let $K$ be a quadratic extension of $k$ or $K=k \times k$. We call a subring of $K$ with 1 an order of $K$ if it is a free $\mathbb{Z}$-module of rank $2 n$. In this paper, we consider orders of $K$ with $\mathfrak{o}_{k}$-module structure. We call such an order a quadratic order over $\mathfrak{o}_{k}$. We shall study the following Dirichlet series:

$$
Z_{k}(s)=|d|^{2 s} \sum_{\mathfrak{O}}|D(\mathfrak{O})|^{-s},
$$

where $\mathfrak{O}$ runs over all quadratic orders over $\mathfrak{o}_{k}$ and $D(\mathfrak{O})$ is the absolute discriminant of the order $\mathfrak{O}$. If $k=\mathbb{Q}$, then it is easy to see that

$$
Z_{\mathbb{Q}}(s)=\left(1-2^{-s}+2^{1-2 s}\right) \zeta(s),
$$

where $\zeta(s)$ is the Riemann zeta function. The purpose of this paper is to generalize this formula to an arbitrary number field $k$. We shall describe $Z_{k}(s)$ in terms of the partial zeta functions of $k$. As an application, we shall give another proof of the density formula for the quadratic extensions of $k$, which was established by D. J. Wright (see Theorem 4.2 of [1]).

1. Quadratic orders. In this section, we shall study the structure of quadratic orders over $\mathfrak{o}_{k}$. The structure of finitely generated $\mathfrak{o}_{k}$-modules is well known. We need the following three lemmas (see, for example, Narkiewicz [3], Chapter 1, §3).

Lemma 1.1. Every non-zero fractional ideal of $k$ is a projective $\mathfrak{o}_{k^{-}}$ module.

Lemma 1.2. Let $A$ be a finitely generated torsion free $\mathfrak{o}_{k}$-module. Then there exists a fractional ideal $\mathfrak{a}$ of $k$ and an integer $m \geq 0$ such that

$$
A \cong \mathfrak{o}_{k}^{m} \oplus \mathfrak{a}
$$

as $\mathfrak{o}_{k}$-modules 
Lemma 1.3. Let $I_{i}, J_{j}(1 \leq i \leq l, 1 \leq j \leq m)$ be non-zero fractional ideals of $k$ and put

$$
A_{1}=I_{1} \oplus \ldots \oplus I_{l}, \quad A_{2}=J_{1} \oplus \ldots \oplus J_{m} .
$$

Then $A_{1}$ and $A_{2}$ are isomorphic if and only if $l=m$ and $I_{1} \ldots I_{l}=$ (a) $J_{1} \ldots J_{m}$ for some $a \in k$.

Let $\mathfrak{O}$ be a quadratic order over $\mathfrak{o}_{k}$. Since $\mathfrak{O}$ is an $\mathfrak{o}_{k}$-module and contains 1 , we have the inclusion $\mathfrak{o}_{k} \subset \mathfrak{O}$. Hence we have the following short exact sequence of $\mathfrak{o}_{k}$-modules:

$$
0 \rightarrow \mathfrak{o}_{k} \rightarrow \mathfrak{O} \rightarrow \mathfrak{O} / \mathfrak{o}_{k} \rightarrow 0 .
$$

Since $\mathfrak{O} / \mathfrak{o}_{k}$ is a finitely generated torsion free $\mathfrak{o}_{k}$-module, Lemma 1.2 implies that

$$
\mathfrak{O} / \mathfrak{o}_{k} \cong \mathfrak{o}_{k}^{m} \oplus \mathfrak{b}
$$

for some fractional ideal $\mathfrak{b}$ of $k$ and some integer $m \geq 0$. Computing the ranks of these modules over $\mathbb{Z}$, we have $m=0$ and $\mathfrak{O} / \mathfrak{o}_{k} \cong \mathfrak{b}$. We denote by $g$ and $\pi$ the isomorphism of $\mathfrak{b}$ onto $\mathfrak{O} / \mathfrak{o}_{k}$ and the natural homomorphism of $\mathfrak{O}$ onto $\mathfrak{O} / \mathfrak{o}_{k}$, respectively. Lemma 1.3 implies that the ideal class of $\mathfrak{b}$ is uniquely determined by $\mathfrak{O}$. It also implies that we may assume $\mathfrak{o}_{k} \subset \mathfrak{b}$. By Lemma $1.1, \mathfrak{b}$ is a projective $\mathfrak{o}_{k}$-module. Hence there exists a homomorphism $f: \mathfrak{O} / \mathfrak{o}_{k} \rightarrow \mathfrak{O}$ such that $\pi \cdot f=$ id. Put $\theta=f \cdot g(1)$. Then we have

$$
\mathfrak{O}=\mathfrak{o}_{k}+\mathfrak{b} \theta \quad \text { (direct sum). }
$$

Since $\mathfrak{O}$ is a ring, $\theta$ satisfies a quadratic equation

$$
q_{a}(x)=x^{2}+a_{1} x+a_{2}=0,
$$

where $a=\left(a_{1}, a_{2}\right) \in \mathfrak{b} \times \mathfrak{o}_{k}$. For any $t, s \in \mathfrak{b}$, we have $t \theta, s \theta \in \mathfrak{O}$. Hence

$$
(t \theta)(s \theta)=-a_{2} t s-a_{1} t s \theta \in \mathfrak{O} .
$$

This implies that $a_{1} \in \mathfrak{b}^{-1}$ and $a_{2} \in \mathfrak{b}^{-2}$. We note that $\mathfrak{b}^{-1}$ is an integral ideal since $\mathfrak{o}_{k} \subset \mathfrak{b}$. If $\mathfrak{O}$ is an order of a quadratic extension of $k$, then $q_{a}(x)$ is irreducible over $k$. If $\mathfrak{O}$ is an order of $k \times k$, then $q_{a}(x)$ is reducible over $k$ with two distinct roots.

Let $\mathfrak{a}$ be an integral ideal of $k$ and let $a=\left(a_{1}, a_{2}\right) \in \mathfrak{a} \times \mathfrak{a}^{2}$. If $q_{a}(x)$ is irreducible over $k$, then we define an associated $\boldsymbol{o}_{k}$-module by

$$
\mathfrak{O}(\mathfrak{a}, a)=\mathfrak{o}_{k}+\mathfrak{a}^{-1} \theta \subset k(\theta),
$$

where $\theta$ is a root of the quadratic equation (1.1). If $q_{a}(x)$ is reducible over $k$ with two distinct roots $\theta_{1}, \theta_{2}$, then we consider the $\boldsymbol{o}_{k}$-module

$$
\mathfrak{D}(\mathfrak{a}, a)=\mathfrak{o}_{k} e+\mathfrak{a}^{-1} \theta \subset k \times k,
$$

where $e=(1,1)$ and $\theta=\left(\theta_{1}, \theta_{2}\right) \in \mathfrak{o}_{k} \times \mathfrak{o}_{k}$. It is easy to see that $\mathfrak{O}(\mathfrak{a}, a)$ is a quadratic order over $\mathfrak{o}_{k}$ in both cases. 
We say that two quadratic orders $\mathfrak{O}_{1}, \mathfrak{O}_{2}$ over $\boldsymbol{o}_{k}$ are $\boldsymbol{o}_{k}$-isomorphic if there exists a ring isomorphism of $\mathfrak{O}_{1}$ onto $\mathfrak{O}_{2}$ which is trivial on $\mathfrak{o}_{k}$. Let $a=\left(a_{1}, a_{2}\right), b=\left(b_{1}, b_{2}\right) \in \mathfrak{a} \times \mathfrak{a}^{2}$. Assume that there exists an $\mathfrak{o}_{k^{-}}$ isomorphism

$$
f: \mathfrak{O}(\mathfrak{a}, b) \rightarrow \mathfrak{O}(\mathfrak{a}, a) .
$$

Then $f\left(\theta_{b}\right)=t \theta_{a}-s$ for some $t \in \mathfrak{a}^{-1}$ and $s \in \mathfrak{o}_{k}$, where $\theta_{a}$ and $\theta_{b}$ are the $\theta$ for $\mathfrak{O}(\mathfrak{a}, a)$ and $\mathfrak{O}(\mathfrak{a}, b)$, respectively. Since $f$ is an $\mathfrak{o}_{k}$-isomorphism, we have

$$
\mathfrak{o}_{k}+\mathfrak{a}^{-1}\left(t \theta_{a}-s\right)=\mathfrak{o}_{k}+\mathfrak{a}^{-1} \theta_{a} .
$$

This implies that $t \in E$ and $s \in \mathfrak{a}$. Further, we have

$$
\begin{gathered}
\left(t^{-1} \theta_{b}+t^{-1} s\right)^{2}+a_{1}\left(t^{-1} \theta_{b}+t^{-1} s\right)+a_{2}=0, \\
\theta_{b}^{2}+\left(2 s+t a_{1}\right) \theta_{b}+\left(s^{2}+a_{1} s t+a_{2} t^{2}\right)=0 .
\end{gathered}
$$

Hence

$$
b_{1}=2 s+t a_{1}, \quad b_{2}=s^{2}+a_{1} s t+a_{2} t^{2} .
$$

So far we have shown the following proposition.

Proposition 1.1. Let $\mathfrak{a}_{i}(i=1, \ldots, h)$ be a complete set of representatives of the ideal classes of $k$, consisting of integral ideals. Then any quadratic order $\mathfrak{O}$ over $\mathfrak{o}_{k}$ is $\mathfrak{a}_{k}$-isomorphic to $\mathfrak{O}\left(\mathfrak{a}_{i}, a\right)$ for some $i$ and some $a \in \mathfrak{a}_{i} \times \mathfrak{a}_{i}^{2}$. Further, $\mathfrak{D}\left(\mathfrak{a}_{i}, a\right) \cong \mathfrak{O}\left(\mathfrak{a}_{j}, b\right)$ if and only if $i=j, b_{1}=2 s+a_{1} t$ and $b_{2}=s^{2}+a_{1} s t+a_{2} t^{2}$ for some $t \in E$ and some $s \in \mathfrak{a}_{i}$.

Let $\mathfrak{a}$ be an integral ideal of $k$. We denote by $E(\mathfrak{a})$ the subgroup of $E$ consisting of all units $\varepsilon$ with $\varepsilon \equiv 1(\bmod \mathfrak{a})$. For any $a=\left(a_{1}, a_{2}\right) \in \mathfrak{a} \times \mathfrak{a}^{2}$, put

$$
\begin{gathered}
Q_{a}(x, y)=y^{2} q_{a}(x / y)=x^{2}+a_{1} x y+a_{2} y^{2}, \\
\Delta\left(Q_{a}\right)=a_{1}^{2}-4 a_{2}
\end{gathered}
$$

and denote by $\mathcal{Q}(\mathfrak{a})$ the set of all $Q_{a}(x, y)\left(a \in \mathfrak{a} \times \mathfrak{a}^{2}\right)$ with $\Delta\left(Q_{a}\right) \neq 0$. We denote by $G(\mathfrak{a})$ (resp. $\left.G^{\prime}(\mathfrak{a})\right)$ the subgroup of $\mathrm{GL}_{2}\left(\boldsymbol{o}_{k}\right)$ consisting of all matrices of the form

$$
\left.\gamma=\left(\begin{array}{ll}
1 & 0 \\
s & t
\end{array}\right), \quad s \in \mathfrak{a}, t \in E \text { (resp. } t \in E(2)\right) .
$$

The action of $\gamma$ on $\mathcal{Q}(\mathfrak{a})$ is defined by

$$
\left(\gamma \cdot Q_{a}\right)(x, y)=Q_{a}(x+s y, t y) .
$$

We denote by $\left[Q_{a}\right]$ (resp. $\left.\left[Q_{a}\right]^{\prime}\right)$ the $G(\mathfrak{a})$-equivalence (resp. $G^{\prime}(\mathfrak{a})$-equivalence) class of $Q_{a}$. Then we can rewrite the previous proposition as follows:

Proposition 1.2. Let $\mathfrak{a}_{i}(i=1, \ldots, h)$ be as in Proposition 1.1. Then the mapping $Q_{a} \mapsto \mathfrak{O}\left(\mathfrak{a}_{i}, a\right)$ induces a bijection of $\bigcup_{i=1}^{h} G\left(\mathfrak{a}_{i}\right) \backslash \mathcal{Q}\left(\mathfrak{a}_{i}\right)$ onto the set of $\mathfrak{o}_{k}$-isomorphism classes of quadratic orders over $\mathfrak{o}_{k}$. 
Now we determine a complete set of representatives for $G(\mathfrak{a}) \backslash \mathcal{Q}(\mathfrak{a})$. Take a complete set of representatives $\alpha_{1}, \ldots, \alpha_{2^{n}} \in \mathfrak{a}-\{0\}$ of the quotient module $\mathfrak{a} / 2 \mathfrak{a}$ and put

$$
\mathcal{Q}\left(\mathfrak{a}, \alpha_{j}\right)=\left\{Q_{a}(x, y) \in \mathcal{Q}(\mathfrak{a}): a_{1} \equiv \alpha_{j}(\bmod 2 \mathfrak{a})\right\} \quad\left(j=1, \ldots, 2^{n}\right) .
$$

Then $\mathcal{Q}(\mathfrak{a})=\bigcup_{j=1}^{2^{n}} \mathcal{Q}\left(\mathfrak{a}, \alpha_{j}\right)$ (disjoint union). It is obvious that the subgroup $G^{\prime}(\mathfrak{a})$ acts on each $\mathcal{Q}\left(\mathfrak{a}, \alpha_{j}\right)$.

LEMMA 1.4. Each $G(\mathfrak{a})$-equivalence class of $\mathcal{Q}(\mathfrak{a})$ consists of exactly $[E: E(2)] G^{\prime}(\mathfrak{a})$-equivalence classes.

Proof. If $E=\bigcup_{\nu=1}^{l} t_{\nu} E(2)$, then $G(\mathfrak{a})=\bigcup_{\nu=1}^{l} G^{\prime}(\mathfrak{a}) \gamma_{\nu}$, where

$$
\gamma_{\nu}=\left(\begin{array}{cc}
1 & 0 \\
0 & t_{\nu}
\end{array}\right)
$$

Hence for any $Q_{a} \in \mathcal{Q}(\mathfrak{a})$, we have $\left[Q_{a}\right]=\bigcup_{\nu=1}^{l}\left[\gamma_{\nu} \cdot Q_{a}\right]^{\prime}$. Assume $\left[\gamma_{\mu} \cdot Q_{a}\right]^{\prime}=$ $\left[\gamma_{\nu} \cdot Q_{a}\right]^{\prime}$. Then $\Delta\left(\gamma_{\mu} \cdot Q_{a}\right)=u^{2} \Delta\left(\gamma_{\nu} \cdot Q_{a}\right)$ for some $u \in E(2)$. This implies that $t_{\mu} / t_{\nu}= \pm u \in E(2)$. Hence $\mu=\nu$.

Lemma 1.5. Let $Q_{a}, Q_{b} \in \mathcal{Q}(\mathfrak{a})$. Then $Q_{a}$ is $G^{\prime}(\mathfrak{a})$-equivalent to $Q_{b}$ if and only if $Q_{a}, Q_{b} \in \mathcal{Q}\left(\mathfrak{a}, \alpha_{j}\right)$ for some $j$ and $\Delta\left(Q_{b}\right)=t^{2} \Delta\left(Q_{a}\right)$ for some $t \in E(2)$.

Proof. The necessity is obvious. To prove the sufficiency, assume $Q_{a}, Q_{b} \in \mathcal{Q}\left(\mathfrak{a}, \alpha_{j}\right)$ for some $j$ and $\Delta\left(Q_{b}\right)=t^{2} \Delta\left(Q_{a}\right)$ for some $t \in E(2)$. Put $s=\left(b_{1}-t a_{1}\right) / 2$. Then we have

$$
2 s=\left(b_{1}-\alpha_{j}\right)+\left(\alpha_{j}-a_{1}\right)+(1-t) a_{1} \in 2 \mathfrak{a},
$$

hence $s \in \mathfrak{a}$ and $b_{1}=t a_{1}+2 s$. Since $\Delta\left(Q_{b}\right)=t^{2} \Delta\left(Q_{a}\right)$, we have

$$
b_{2}=\left\{b_{1}^{2}-t^{2}\left(a_{1}^{2}-4 a_{2}\right)\right\} / 4=s^{2}+a_{1} s t+a_{2} t^{2} .
$$

Hence $\gamma \cdot Q_{a}=Q_{b}$, where $\gamma$ is an element of $G^{\prime}(\mathfrak{a})$ defined by (1.2).

Proposition 1.3. The mapping $Q_{a} \mapsto \Delta\left(Q_{a}\right)$ induces a bijection

$$
G^{\prime}(\mathfrak{a}) \backslash \mathcal{Q}\left(\mathfrak{a}, \alpha_{j}\right) \leftrightarrow\left(\alpha_{j}^{2}+4 \mathfrak{a}^{2}\right)^{\prime} / E(2)^{2},
$$

where $\left(\alpha_{j}^{2}+4 \mathfrak{a}^{2}\right)^{\prime}=\left(\alpha_{j}^{2}+4 \mathfrak{a}^{2}\right)-\{0\} \subset k$.

Pr o of. Lemma 1.5 implies the injectivity of the mapping. The surjectivity is obvious.

For any integral ideal $\mathfrak{a}$ of $k$, we denote by $N(\mathfrak{a})$ the absolute norm of $\mathfrak{a}$. Then the following formula is easily deduced from the definition of the discriminant.

LEMMA 1.6 .

$$
D(\mathfrak{O}(\mathfrak{a}, a))=d^{2} N\left(\Delta\left(Q_{a}\right) \mathfrak{a}^{-2}\right) .
$$


This lemma implies that $D(\mathfrak{O}) d^{-2}$ is a rational integer for any quadratic order $\mathfrak{O}$ over $\mathfrak{o}_{k}$. We denote it by $D\left(\mathfrak{O} ; \mathfrak{o}_{k}\right)$. For any quadratic extension $K$ of $k$, we denote by $\mathfrak{O}_{K}$ the ring of integers of $K$. Then we have

$$
\left|D\left(\mathfrak{O}_{K} ; \mathfrak{o}_{k}\right)\right|=N\left(D_{K / k}\right),
$$

where $D_{K / k}$ is the relative discriminant of the quadratic extension $K / k$. Using this notation, the Dirichlet series $Z_{k}(s)$ is written as follows:

$$
Z_{k}(s)=\sum_{\mathfrak{O}}\left|D\left(\mathfrak{O} ; \mathfrak{o}_{k}\right)\right|^{-s} .
$$

Now it follows from Propositions 1.2, 1.3, Lemma 1.4 and the above equation (1.3) that

$$
\begin{aligned}
Z_{k}(s) & =\sum_{i=1}^{h} \sum_{Q \in G\left(\mathfrak{a}_{i}\right) \backslash \mathcal{Q}\left(\mathfrak{a}_{i}\right)} N\left(\Delta(Q) \mathfrak{a}_{i}^{-2}\right)^{-s} \\
& =\frac{1}{[E: E(2)]} \sum_{i=1}^{h} \sum_{Q \in G^{\prime}\left(\mathfrak{a}_{i}\right) \backslash \mathcal{Q}\left(\mathfrak{a}_{i}\right)} N\left(\Delta(Q) \mathfrak{a}_{i}^{-2}\right)^{-s} \\
& =\frac{1}{[E: E(2)]} \sum_{i=1}^{h} \sum_{j=1}^{2^{n}} \sum_{Q \in G^{\prime}\left(\mathfrak{a}_{i}\right) \backslash \mathcal{Q}\left(\mathfrak{a}_{i}, \alpha_{i j}\right)} N\left(\Delta(Q) \mathfrak{a}_{i}^{-2}\right)^{-s} \\
& =\frac{1}{[E: E(2)]} \sum_{i=1}^{h} \sum_{j=1}^{2^{n}} \sum_{\delta \in\left(\alpha_{i j}^{2}+4 \mathfrak{a}_{i}^{2}\right)^{\prime} / E(2)^{2}} N\left((\delta) \mathfrak{a}_{i}^{-2}\right)^{-s},
\end{aligned}
$$

where $\left(\alpha_{i j}^{2}+4 \mathfrak{a}_{i}^{2}\right)^{\prime}=\left(\alpha_{i j}^{2}+4 \mathfrak{a}_{i}^{2}\right)-\{0\}$. To calculate the innermost sum, we shall introduce the partial zeta functions of $k$ in the next section.

2. Dirichlet series of discriminants of quadratic orders. We use the same notations as in the previous section. Let $r_{1}, r_{2}$ be the numbers of real and imaginary primes of $k$, respectively. Hence $r_{1}+2 r_{2}=n$. We denote by $M_{r}$ the set of real primes of $k$. If $v \in M_{r}$, we denote by $\sigma_{v}$ the corresponding embedding of $k$ into $\mathbb{R}$. For any subset $S$ of $M_{r}$, we denote by $\mathfrak{h}_{S}$ the product of the real primes in $S$. Further, we denote by $k_{S}$ the subset of $k^{\times}$consisting of all elements $\gamma \in k^{\times}$satisfying $\sigma_{v}(\gamma)>0$ for all $v \in S$. For any subset $A$ of $k$, put $A_{S}=A \cap k_{S}$. If $\mathfrak{O}=\mathfrak{O}(\mathfrak{a}, a)$ is a quadratic order over $\mathfrak{o}_{k}$, then $\Delta(\mathfrak{O})=\Delta\left(Q_{a}\right)$ is determined up to multiplication by an element of $E^{2}$. Using these notations, we define the Dirichlet series $Z_{k, S}(s)$ as follows:

$$
Z_{k, S}(s)=\sum_{\mathfrak{O}}\left|D\left(\mathfrak{O} ; \mathfrak{o}_{k}\right)\right|^{-s},
$$


where $\mathfrak{O}$ runs over all quadratic orders over $\mathfrak{o}_{k}$ with $\Delta(\mathfrak{O}) \in k_{S}$. We note that if $S$ is the empty set, then $Z_{k, S}(s)$ coincides with $Z_{k}(s)$.

Let $\mathfrak{f}$ be a non-zero integral ideal of $k$. We denote by $I(\mathfrak{f})$ the multiplicative group consisting of all non-zero fractional ideals of $k$ which are relatively prime to $\mathfrak{f}$. We denote by $P\left(\mathfrak{f} \mathfrak{h}_{S}\right)$ the subgroup of $I(\mathfrak{f})$ consisting of all principal fractional ideals $(\delta)$ with $\delta \in k_{S}$ and $\delta \equiv 1\left(\bmod ^{*} \mathfrak{f}\right)$. Here $\delta \equiv 1\left(\bmod ^{*} \mathfrak{f}\right)$ means that $\delta=\alpha / \beta$ for some $\alpha, \beta \in \mathfrak{o}_{k}$ satisfying $(\alpha \beta, \mathfrak{f})=1$ and $\alpha \equiv \beta(\bmod \mathfrak{f})$. We call the quotient group $I(\mathfrak{f}) / P\left(\mathfrak{f h}_{S}\right)$ the group of ray classes modulo $\mathfrak{f h}_{S}$ and denote it by $H\left(\mathfrak{f} \mathfrak{h}_{S}\right)$. For any $\mathfrak{b} \in I(\mathfrak{f})$, we denote by $\left[\mathfrak{b}, \mathfrak{f h}_{S}\right]$ the class in $H\left(\mathfrak{f h}_{S}\right)$ represented by $\mathfrak{b}$. Now the partial zeta function of $c \in H\left(\mathfrak{f h}_{S}\right)$ is defined by

$$
\zeta_{k, \mathfrak{f h}}(s, c)=\sum_{\mathfrak{b}} N(\mathfrak{b})^{-s}
$$

where $\mathfrak{b}$ runs over all integral ideals belonging to the ray class $c$. We need some lemmas to give an expression of our Dirichlet series $Z_{k, S}(s)$ in terms of the partial zeta functions of $k$. We put $E\left(\mathfrak{f} \mathfrak{h}_{S}\right)=E(\mathfrak{f}) \cap k_{S}$.

LEMMA 2.1. Let $\mathfrak{a}$ be a non-zero integral ideal of $k$ and let $\alpha$ be a nonzero element of $\mathfrak{a}$. Put $\mathfrak{g}=\left(\alpha \mathfrak{a}^{-1}, 2\right)$ and $\mathfrak{f}=(2) \mathfrak{g}^{-1}$. Then

$$
\sum_{\delta \in\left(\alpha^{2}+4 \mathfrak{a}^{2}\right)_{S} / E(2)^{2}} N\left((\delta) \mathfrak{a}^{-2}\right)^{-s}=\left[E\left(\mathfrak{f}^{2} \mathfrak{h}_{S}\right): E(2)^{2}\right] N(\mathfrak{g})^{-2 s} \zeta_{k, \mathfrak{f}^{2} \mathfrak{h}_{S}}\left(s, c^{2}\right),
$$

where $c$ is the ray class in $H\left(\mathfrak{f}^{2} \mathfrak{h}_{S}\right)$ represented by the integral ideal $(\alpha) \mathfrak{a}^{-1} \mathfrak{g}^{-1}$

Proof. Let $\delta \in\left(\alpha^{2}+4 \mathfrak{a}^{2}\right)_{S}$ and put $\mathfrak{b}=(\delta) \mathfrak{a}^{-2} \mathfrak{g}^{-2}$. Then $\mathfrak{b}$ is an integral ideal prime to $\mathfrak{f}$. Since $\delta \alpha^{-2} \equiv 1\left(\bmod ^{*} \mathfrak{f}^{2}\right)$ and $\delta \alpha^{-2} \in k_{S}$, the integral ideal $\mathfrak{b}$ belongs to the ray class $c^{2}$. Conversely, if $\mathfrak{b}$ is an integral ideal belonging to the ray class $c^{2}$, then $\mathfrak{b}=(\beta)\left(\alpha^{2}\right) \mathfrak{a}^{-2} \mathfrak{g}^{-2}$ for some $\beta \in k_{S}$ with $\beta \equiv 1\left(\bmod ^{*} \mathfrak{f}^{2}\right)$. Hence $\mathfrak{b}=(\delta) \mathfrak{a}^{-2} \mathfrak{g}^{-2}$ with $\delta=\beta \alpha^{2} \in\left(\alpha^{2}+4 \mathfrak{a}^{2}\right)_{S}$. Let $\delta_{1}, \delta_{2} \in\left(\alpha^{2}+4 \mathfrak{a}^{2}\right)_{S}$ and denote by $\mathfrak{b}_{1}$ and $\mathfrak{b}_{2}$ the ideals corresponding to $\delta_{1}$ and $\delta_{2}$, respectively. Then $\mathfrak{b}_{1}=\mathfrak{b}_{2}$ if and only if $\delta_{1} / \delta_{2} \in E\left(\mathfrak{f}^{2} \mathfrak{h}_{S}\right)$. Now the desired formula follows immediately.

For any $\mathfrak{f} \mid 2$, and for any fractional ideal $\mathfrak{c}$ of $k$ relatively prime to $\mathfrak{f}$, put

$$
\varrho_{\mathfrak{f}}([\mathfrak{c}, \mathfrak{f}])=\left[\mathfrak{c}^{2}, \mathfrak{f}^{2} \mathfrak{h}_{S}\right] .
$$

Then $\varrho_{\mathfrak{f}}$ is a well defined homomorphism of $H(\mathfrak{f})$ to $H\left(\mathfrak{f}^{2} \mathfrak{h}_{S}\right)$.

LEMMA 2.2. If the order of the group $H\left(\mathfrak{f}^{2} \mathfrak{h}_{S}\right)$ is odd, then $\varrho_{\mathfrak{f}}$ is an isomorphism of $H(\mathfrak{f})$ onto $H\left(\mathfrak{f}^{2} \mathfrak{h}_{S}\right)$.

Proof. The assumption implies that any element of $H\left(\mathfrak{f}^{2} \mathfrak{h}_{S}\right)$ can be written as $\left[\mathfrak{c}, \mathfrak{f}^{2} \mathfrak{h}_{S}\right]^{2}$ for some ideal $\mathfrak{c}$. Hence $\varrho_{\mathfrak{f}}$ is surjective. On the other 
hand, we have the natural surjective homomorphism of $H\left(\mathfrak{f}^{2} \mathfrak{h}_{S}\right)$ onto $H(\mathfrak{f})$. Hence $\varrho_{\mathfrak{f}}$ must be an isomorphism.

The order of the group $H\left(\mathfrak{f h} \mathfrak{h}_{S}\right)$ is given by the following lemma (see Lang [2], Chapter VI, Theorem 1).

LEMMA 2.3. For any non-zero integral ideal $\mathfrak{f}$,

$$
\# H\left(\mathfrak{f} \mathfrak{h}_{S}\right)=\frac{h \varphi(\mathfrak{f}) 2^{\# S}}{\left[E: E\left(\mathfrak{f} \mathfrak{h}_{S}\right)\right]},
$$

where $\varphi$ is the Euler function of $k$.

Now we are ready to prove our main theorem.

\section{THEOREM 1.}

$$
Z_{k, S}(s)=2^{r_{1}+r_{2}-2 n s} \sum_{\mathfrak{f} \mid 2} \frac{N(\mathfrak{f})^{2 s}}{\left[E(\mathfrak{f}): E\left(\mathfrak{f}^{2} \mathfrak{h}_{S}\right)\right]} \sum_{c \in H(\mathfrak{f})} \zeta_{k, \mathfrak{f}^{2} \mathfrak{h}_{S}}\left(s, \varrho_{\mathfrak{f}}(c)\right) .
$$

In particular, if the order of $H\left(4 \mathfrak{h}_{S}\right)$ is odd, then

$$
Z_{k, S}(s)=2^{r_{1}+r_{2}-\# S-2 n s} \zeta_{k}(s) \sum_{\mathfrak{f} \mid 2} N(\mathfrak{f})^{2 s-1} \prod_{\mathfrak{p} \mid \mathfrak{f}}\left(1-N(\mathfrak{p})^{-s}\right),
$$

where $\zeta_{k}(s)$ is the Dedekind zeta function of $k$.

Proof. Let $\alpha_{i 1}, \ldots, \alpha_{i 2^{n}} \in \mathfrak{a}_{i}-\{0\}$ be a complete set of representatives of the quotient module $\mathfrak{a}_{i} / 2 \mathfrak{a}_{i}$. Then in the same way as when deducing the equation (1.4), we get

$$
Z_{k, S}(s)=\frac{1}{[E: E(2)]} \sum_{i=1}^{h} \sum_{j=1}^{2^{n}} \sum_{\delta \in\left(\alpha_{i j}^{2}+4 \mathfrak{a}_{i}^{2}\right)_{S} / E(2)^{2}} N\left((\delta) \mathfrak{a}_{i}^{-2}\right)^{-s} .
$$

By Lemma 2.1 and the above equation (2.3), we have

$$
Z_{k, S}(s)=\sum_{i=1}^{h} \sum_{j=1}^{2^{n}} \frac{\left[E\left(\mathfrak{f}^{2} \mathfrak{h}_{S}\right): E(2)^{2}\right]}{[E: E(2)]} N(\mathfrak{g})^{-2 s} \zeta_{k, \mathfrak{f}^{2} \mathfrak{h}_{S}}\left(s, \varrho_{\mathfrak{f}}\left(c_{i j}\right)\right)
$$

where $\mathfrak{g}=\left(\alpha_{i j} \mathfrak{a}_{i}^{-1}, 2\right), \mathfrak{f}=(2) \mathfrak{g}^{-1}$ and $c_{i j}=\left[\left(\alpha_{i j}\right) \mathfrak{a}_{i}^{-1} \mathfrak{g}^{-1}, \mathfrak{f}\right]$. For any $\mathfrak{g} \mid 2$, we consider the sum

$$
T_{\mathfrak{g}}=\sum \zeta_{k, \mathfrak{f}^{2}}\left(s, c_{i j}^{2}\right)
$$

where the summation is taken over all $i, j$ with $\left(\alpha_{i j} \mathfrak{a}_{i}^{-1}, 2\right)=\mathfrak{g}$. Then

$$
Z_{k, S}(s)=\sum_{\mathfrak{g} \mid 2} \frac{\left[E\left(\mathfrak{f}^{2} \mathfrak{h}_{S}\right): E(2)^{2}\right]}{[E: E(2)]} N(\mathfrak{g})^{-2 s} T_{\mathfrak{g}} .
$$

Now we claim that the ray class $c_{i j}$ in $T_{\mathfrak{g}}$ represents every element of $H(\mathfrak{f})$ exactly $[E: E(\mathfrak{f})]$ times. To prove this, for any $c \in H(\mathfrak{f})$, take an integral ideal $\mathfrak{b}$ relatively prime to $\mathfrak{f}$ such that $c=[\mathfrak{b}, \mathfrak{f}]$. Since $\mathfrak{a}_{1}^{-1} \mathfrak{g}^{-1}, \ldots, \mathfrak{a}_{h}^{-1} \mathfrak{g}^{-1}$ is 
a complete set of representatives of the ideal classes of $k, \mathfrak{b}=(\gamma) \mathfrak{a}_{i}^{-1} \mathfrak{g}^{-1}$ for some $i$ and $\gamma \in k^{\times}$. Since $\mathfrak{b}$ is integral, we have $\gamma \in \mathfrak{a}_{i} \mathfrak{g} \subset \mathfrak{a}_{i}$. Hence $\gamma \equiv \alpha_{i j}$ $\left(\bmod 2 \mathfrak{a}_{i}\right)$ for some $j$. Now the fact that $(\mathfrak{b}, \mathfrak{f})=1$ implies $\left(\alpha_{i j} \mathfrak{a}_{i}^{-1}, 2\right)=\mathfrak{g}$ and $\gamma \alpha_{i j}^{-1} \equiv 1\left(\bmod ^{*} \mathfrak{f}\right)$. Hence $[\mathfrak{b}, \mathfrak{f}]=c_{i j}$. It is easy to see that $c_{i^{\prime} j^{\prime}}=c_{i j}$ if and only if $i^{\prime}=i$ and $\alpha_{i^{\prime} j^{\prime}} \alpha_{i j}^{-1} \equiv \varepsilon\left(\bmod ^{*} \mathfrak{f}\right)$ for some $\varepsilon \in E$. This proves our claim, and hence we have established the following equation:

$$
T_{\mathfrak{g}}=[E: E(\mathfrak{f})] \sum_{c \in H(\mathfrak{f})} \zeta_{k, \mathfrak{f}^{2} \mathfrak{h}_{S}}\left(s, \varrho_{\mathfrak{f}}(c)\right) .
$$

It is clear that

$$
\frac{\left[E\left(\mathfrak{f}^{2} \mathfrak{h}_{S}\right): E(2)^{2}\right][E: E(\mathfrak{f})]}{[E: E(2)]}=\frac{\left[E(2): E(2)^{2}\right]}{\left[E(\mathfrak{f}): E\left(\mathfrak{f}^{2} \mathfrak{h}_{S}\right)\right]} .
$$

Dirichlet's unit theorem and the fact that $\pm 1 \in E(2)$ imply

$$
\left[E(2): E(2)^{2}\right]=2^{r_{1}+r_{2}} \text {. }
$$

By (2.4)-(2.7), we have

$$
\begin{aligned}
Z_{k, S}(s) & =2^{r_{1}+r_{2}} \sum_{\mathfrak{g} \mid 2} \frac{N(\mathfrak{g})^{-2 s}}{\left[E(\mathfrak{f}): E\left(\mathfrak{f}^{2} \mathfrak{h}_{S}\right)\right]} \sum_{c \in H(\mathfrak{f})} \zeta_{k, \mathfrak{f}^{2} \mathfrak{h}_{S}}\left(s, \varrho_{\mathfrak{f}}(c)\right) \\
& =2^{r_{1}+r_{2}-2 n s} \sum_{\mathfrak{f} \mid 2} \frac{N(\mathfrak{f})^{2 s}}{\left[E(\mathfrak{f}): E\left(\mathfrak{f}^{2} \mathfrak{h}_{S}\right)\right]} \sum_{c \in H(\mathfrak{f})} \zeta_{k, \mathfrak{f}^{2} \mathfrak{h}_{S}}\left(s, \varrho_{\mathfrak{f}}(c)\right) .
\end{aligned}
$$

Now we assume that the order of $H\left(4 \mathfrak{h}_{S}\right)$ is odd. Then the order of $H\left(\mathfrak{f}^{2} \mathfrak{h}_{S}\right)$ is odd for any $\mathfrak{f} \mid 2$. By Lemma 2.2, $\varrho_{\mathfrak{f}}$ is an isomorphism of $H(\mathfrak{f})$ onto $H\left(\mathfrak{f}^{2} \mathfrak{h}_{S}\right)$. Hence the inner sum of the right hand side of $(2.8)$ is equal to

$$
\sum_{c \in H\left(\mathfrak{f}^{2} \mathfrak{h}_{S}\right)} \zeta_{k, \mathfrak{f}^{2} \mathfrak{h}_{S}}(s, c)=\zeta_{k}(s) \prod_{\mathfrak{p} \mid \mathfrak{f}}\left(1-N(\mathfrak{p})^{-s}\right) .
$$

On the other hand, the fact that $H(\mathfrak{f}) \cong H\left(\mathfrak{f}^{2} \mathfrak{h}_{S}\right)$ and Lemma 2.3 imply

$$
\left[E(\mathfrak{f}): E\left(\mathfrak{f}^{2} \mathfrak{h}_{S}\right)\right]=2^{\# S} N(\mathfrak{f}) .
$$

Now the second formula of the theorem follows from (2.8)-(2.10).

COROLlary 1. The Dirichlet series $Z_{k, S}(s)$ converges absolutely for $\operatorname{Re} s>1$ and can be analytically continued to a meromorphic function on the whole complex plane. Its only singularity is a simple pole at $s=1$ with residue

$$
\frac{2^{r_{1}-\# S} \pi^{r_{2}} R h}{w \sqrt{|d|}},
$$

where $R$ is the regulator of $k$ and $w$ is the number of roots of unity contained in $k$. 
Proof. The first statement is obvious because the corresponding one for the partial zeta functions holds. It is well known that the residue of the partial zeta function $\zeta_{k, \mathfrak{f}^{2}}(s, c)$ at $s=1$ does not depend on the ray class $c$. Hence

$$
\operatorname{Res}_{s=1} \zeta_{k, \mathfrak{f}^{2} \mathfrak{h}_{S}}(s, c)=\frac{\operatorname{Res}_{s=1} \zeta_{k}(s)}{\# H\left(\mathfrak{f}^{2} \mathfrak{h}_{S}\right)} \prod_{\mathfrak{p} \mid \mathfrak{f}}\left(1-N(\mathfrak{p})^{-1}\right)
$$

By Theorem 1, Lemma 2.3 and the above equation (2.11), we have

$$
\begin{aligned}
\operatorname{Res}_{s=1} Z_{k, S}(s)= & 2^{r_{1}+r_{2}-2 n} \sum_{\mathfrak{f} \mid 2} \frac{N(\mathfrak{f})^{2}}{\left[E(\mathfrak{f}): E\left(\mathfrak{f}^{2} \mathfrak{h}_{S}\right)\right]} \frac{\# H(\mathfrak{f})}{\# H\left(\mathfrak{f}^{2} \mathfrak{h}_{S}\right)} \\
& \times \operatorname{Res}_{s=1} \zeta_{k}(s) \prod_{\mathfrak{p} \mid \mathfrak{f}}\left(1-N(\mathfrak{p})^{-1}\right) \\
= & 2^{r_{1}+r_{2}-\# S-2 n} \operatorname{Res}_{s=1} \zeta_{k}(s) \sum_{\mathfrak{f} \mid 2} \varphi(\mathfrak{f}) \\
= & 2^{-r_{2}-\# S} \operatorname{Res}_{s=1} \zeta_{k}(s) .
\end{aligned}
$$

It is well known that

$$
\operatorname{Res}_{s=1} \zeta_{k}(s)=\frac{2^{r_{1}+r_{2}} \pi^{r_{2}} R h}{w \sqrt{|d|}}
$$

(see, for example, [2], Chapter VIII, Theorem 5). Now the desired formula for the residue of $Z_{k, S}(s)$ at $s=1$ follows from (2.12) and (2.13).

Corollary 1 and the Ikehara theorem imply

Corollary 2.

$$
\#\left\{\mathfrak{O}: \Delta(\mathfrak{O}) \in k_{S},\left|D\left(\mathfrak{O} ; \mathfrak{o}_{k}\right)\right| \leq X\right\} \sim \frac{2^{r_{1}-\# S} \pi^{r_{2}} R h}{w \sqrt{|d|}} X \quad \text { as } X \rightarrow \infty .
$$

Corollary 3. Assume that the order of the group $H\left(4 \mathfrak{h}_{S}\right)$ is odd. Put

$$
\begin{gathered}
A=2^{-r_{1}} \pi^{-n / 2}|d|^{1 / 2}, \\
G_{k, S}(s)=2^{n s} A^{s} \Gamma\left(\frac{s}{2}\right)^{r_{1}} \Gamma(s)^{r_{2}} Z_{k, S}(s) .
\end{gathered}
$$

Then $G_{k, S}(s)$ satisfies the functional equation

$$
G_{k, S}(1-s)=G_{k, S}(s) \text {. }
$$

Proof. Let $(2)=\mathfrak{p}_{1}^{e_{1}} \ldots \mathfrak{p}_{g}^{e_{g}}$ be the prime ideal factorization of 2 in $k$. For any $\mathfrak{f} \mid 2$, put

$$
\psi(s, \mathfrak{f})=N(\mathfrak{f})^{2 s-1} \prod_{\mathfrak{p} \mid \mathfrak{f}}\left(1-N(\mathfrak{p})^{-s}\right)
$$


and

$$
f(s)=\sum_{\mathfrak{f} \mid 2} \psi(s, \mathfrak{f}) .
$$

Since $\psi(s, \mathfrak{f})$ is multiplicative, we have

$$
f(s)=\prod_{i=1}^{g} f_{i}(s),
$$

where $f_{i}(s)=\sum_{r=0}^{e_{i}} \psi\left(s, \mathfrak{p}_{i}^{r}\right)$. Then

$$
f_{i}(1-s)=N\left(\mathfrak{p}_{i}\right)^{e_{i}(1-2 s)} f_{i}(s), \quad i=1, \ldots, g .
$$

Hence $f(s)$ satisfies

$$
f(1-s)=f(s) \prod_{i=1}^{g} N\left(\mathfrak{p}_{i}\right)^{e_{i}(1-2 s)}=f(s) 2^{n(1-2 s)} .
$$

Now the functional equation of the Dedekind zeta function (see [2], Chapter XIII, Theorem 2) and (2.14) imply $G_{k, S}(1-s)=G_{k, S}(s)$.

3. Quadratic extensions. Let $S$ be a subset of $M_{r}$. In this section, we study the following Dirichlet series:

$$
\xi_{k, S}(s)=\sum_{K} N\left(D_{K / k}\right)^{-s}
$$

where $K$ runs over all quadratic extensions of $k$ which are unramified at any $v \in S$. Wright studied this Dirichlet series in [4] and [5] by class field theory and by developing the theory of Iwasawa-Tate zeta function, respectively.

Let $K$ be a quadratic extension of $k$. Then $\mathfrak{O}_{K}$ is a quadratic order over $\mathfrak{o}_{k}$. Hence $\mathfrak{O}_{K}=\mathfrak{O}\left(\mathfrak{a}_{i}, a\right)$ for some $i$ and $a \in \mathfrak{a}_{i} \times \mathfrak{a}_{i}^{2}$. If $\theta$ is a root of the quadratic equation $q_{a}(x)=0$, then $\mathfrak{O}_{K}=\mathfrak{o}_{k}+\mathfrak{a}_{i}^{-1} \theta$. Let $\mathfrak{O}$ be a quadratic order over $\mathfrak{o}_{k}$ contained in $K$. Since $\mathfrak{O} \subset \mathfrak{O}_{K},\left\{\lambda \in \mathfrak{a}_{i}^{-1}: \lambda \theta \in \mathfrak{O}\right\}$ is a fractional ideal of $k$ contained in $\mathfrak{a}_{i}^{-1}$. Hence $\mathfrak{O}$ can be written

$$
\mathfrak{O}=\mathfrak{o}_{k}+\mathfrak{a}_{i}^{-1} \mathfrak{b} \theta
$$

for some integral ideal $\mathfrak{b}$ of $k$. Conversely, the $\mathfrak{o}_{k}$-module defined by (3.1) is obviously a quadratic order over $\mathfrak{o}_{k}$ contained in $K$. Hence

$$
\sum_{\mathfrak{O} \subset K}\left|D\left(\mathfrak{O} ; \mathfrak{o}_{k}\right)\right|^{-s}=\sum_{\mathfrak{b}} N\left(D_{K / k}\right)^{-s} N(\mathfrak{b})^{-2 s}=N\left(D_{K / k}\right)^{-s} \zeta_{k}(2 s) .
$$

Let $K=k \times k$ and denote by $\mathfrak{O}_{K}$ the maximal order of $K$. Then $\mathfrak{O}_{K}=$ $\mathfrak{o}_{k} e+\mathfrak{o}_{k} \theta$ with $e=(1,1)$ and $\theta=(0,1)$. Any quadratic order contained in $K$ is of the form $\mathfrak{o}_{k} e+\mathfrak{b} \theta$ for some integral ideal $\mathfrak{b}$ of $k$. Hence

$$
\sum_{\mathfrak{O} \subset K}\left|D\left(\mathfrak{O} ; \mathfrak{o}_{k}\right)\right|^{-s}=\sum_{\mathfrak{b}} N(\mathfrak{b})^{-2 s}=\zeta_{k}(2 s) .
$$


By Corollary 1, the Dirichlet series $Z_{k}(s)$ converges absolutely for $\operatorname{Re} s>1$. Hence the equations (3.2) and (3.3) imply

$$
Z_{k, S}(s)=\zeta_{k}(2 s)+\zeta_{k}(2 s) \xi_{k, S}(s) .
$$

By (3.4) and Theorem 1, we have given another proof of the following theorem which is a special case of Wright's theorem.

\section{TheOREM 2.}

$$
\xi_{k, S}(s)=\frac{2^{r_{1}+r_{2}-2 n s}}{\zeta_{k}(2 s)} \sum_{\mathfrak{f} \mid 2} \frac{N(\mathfrak{f})^{2 s}}{\left[E(\mathfrak{f}): E\left(\mathfrak{f}^{2} \mathfrak{h}_{S}\right)\right]} \sum_{c \in H(\mathfrak{f})} \zeta_{k, \mathfrak{f}^{2} \mathfrak{h}_{S}}\left(s, \varrho_{\mathfrak{f}}(c)\right)-1 .
$$

Corollary 4. Denote by $c_{S}(X)$ the number of quadratic extensions $K$ of $k$ with $\left|N\left(D_{K / k}\right)\right| \leq X$ which are unramified at any $v \in S$. Then

$$
c_{S}(X) \sim \frac{2^{r_{1}-\# S} \pi^{r_{2}} R h}{w \sqrt{|d|} \zeta_{k}(2)} X \quad \text { as } X \rightarrow \infty .
$$

By Corollary 4 and an elementary argument on counting cardinality, we have

Corollary 5. Denote by $c_{S}^{\prime}(X)$ the number of quadratic extensions $K$ of $k$ with $\left|N\left(D_{K / k}\right)\right| \leq X$ which are unramified at any $v \in S$ and ramified at any $v \in M_{r}-S$. Then

$$
c_{S}^{\prime}(X) \sim \frac{\pi^{r_{2}} R h}{w \sqrt{|d|} \zeta_{k}(2)} X \quad \text { as } X \rightarrow \infty .
$$

\section{References}

[1] B. Datskovsky and D. J. Wright, Density of discriminants of cubic extensions, J. Reine Angew. Math. 386 (1988), 116-138.

[2] S. Lang, Algebraic Number Theory, Addison-Wesley, 1970.

[3] W. Narkiewicz, Elementary and Analytic Theory of Algebraic Numbers, Springer, 1990.

[4] D. J. Wright, Distribution of discriminants of abelian extensions, Proc. London Math. Soc. (3) 58 (1989), 17-50.

[5] -, Twists of the Iwasawa-Tate zeta function, Math. Z. 200 (1989), 209-231.

DEPARTMENT OF MATHEMATICS

JOETSU UNIVERSITY OF EDUCATION

JOETSU 943, JAPAN 